\title{
BMJ Open Prostatectomy versus radiotherapy for early-stage prostate cancer (PREPaRE) study: protocol for a mixed-methods study of treatment decision-making in men with localised prostate cancer
}

\author{
Allan Ben Smith, ${ }^{1,2}$ Pascal Mancuso, ${ }^{2,3}$ Mark Sidhom, ${ }^{2,4}$ Karen Wong, ${ }^{2,4}$ \\ Megan Berry, ${ }^{2,4}$ Orlando Rincones, ${ }^{1}$ Dion Forstner, ${ }^{4,5}$ Lesley Bokey, ${ }^{1,6}$ Afaf Girgis ${ }^{1,2}$
}

To cite: Smith $A B$, Mancuso $P$, Sidhom M, et al. Prostatectomy versus radiotherapy for early-stage prostate cancer (PREPaRE) study: protocol for a mixed-methods study of treatment decisionmaking in men with localised prostate cancer. BMJ Open 2017;7:e018403. doi:10.1136/ bmjopen-2017-018403

- Prepublication history for this paper is available online. To view please visit the journal online (http://dx.doi.org/10. 1136/bmjopen-2017-018403).

Received 28 June 2017 Revised 29 August 2017 Accepted 26 September 2017

\section{CrossMark}

For numbered affiliations see end of article.

Correspondence to

Dr Allan Ben Smith;

ben.smith@unsw.edu.au

\section{ABSTRACT}

Background Men diagnosed with localised prostate cancer (LPC) wanting curative treatment face a highly preferencesensitive choice between prostatectomy and radiotherapy, which offer similar cure rates but different side effects. This study aims to determine the information, decision-making needs and preferences of men with LPC choosing between robotic prostatectomy and standard external beam or stereotactic radiotherapy.

Methods and analysis This study will be conducted at a large public teaching hospital in Australia offering the choice between robotic prostatectomy and radiotherapy from early 2017. Men (20-30) diagnosed with LPC who want curative treatment and meet criteria for either treatment will be invited to participate. In this mixed-methods study, patients will complete semistructured interviews before and after attending a combined clinic in which they consult a urologist and a radiation oncologist regarding treatment and four questionnaires (one before treatment decision-making and three after) assessing demographic and clinical characteristics, involvement in decision-making, decisional conflict, satisfaction and regret. Combined clinic consultations will also be audio-recorded and clinicians will report their perceptions regarding patients' suitability for, openness to and preferences for each treatment. Qualitative data will be transcribed verbatim and thematically analysed and descriptive statistical analyses will explore quantitative decision-making outcomes, with comparison according to treatment choice.

Discussion Results from this study will inform how to best support men diagnosed with LPC deciding which curative treatment option best suits their needs and may identify the need for and content required in a decision aid to support these men

Ethics and dissemination All participants will provide written informed consent. Data will be rigorously managed in accordance with national legislation. Results will be disseminated via presentations to both scientific and layperson audiences and publications in peer-reviewed scientific journals.

\section{INTRODUCTION}

Prostate cancer (PC) is the most commonly diagnosed cancer in Australia. In 2013, 19233

\section{Strengths and limitations of this study}

- This mixed-methods study provides a multifaceted perspective on the information and decision-making needs of men with localised prostate cancer (LPC) choosing between robotic prostatectomy and radiotherapy.

- This adds to the limited research specifically focused on decisions involving robotic prostatectomy as an option, which is critical, given the increased availability and uptake of robotic prostatectomies by men with LPC despite limited evidence of superior efficacy.

- Semistructured patient interviews will be thematically analysed to identify information and decision-making preferences and needs of men with LPC. Patient consultations with both urologists and radiation oncologists will be analysed using the OPTION-5 to see whether these preferences and needs are addressed in consultations.

- Qualitative data will be complemented by quantitative data exploring longitudinal decisionmaking outcomes from before a treatment decision is made to six6 months after treatment completion.

- The small sample size and single study site limit the generalisability of the study results, which will nonetheless provide important preliminary insights into the information and decision making needs of men with LPC deciding between robotic prostatectomy and radiotherapy.

new cases of PC were diagnosed, representing $15 \%$ of all new cancers diagnosed in Australia. ${ }^{1}$ Approximately one in five Australian men will be diagnosed with PC before the age of $85,{ }^{1}$ estimated at approximately 16665 males diagnosed in 2017 alone. ${ }^{2}$ With 5-year survival for men with cancer confined to the prostate (localised prostate cancer (LPC)) being very high at $95 \%,{ }^{2}$ the population of men living with and beyond PC is substantial 
and growing. The impact of PC treatments on these men's quality of life can be experienced for many years post-treatment; ${ }^{34}$ hence, it is imperative that treatment choices are well understood and supported.

\section{Treatment options for localised prostate cancer}

Treatment options for men diagnosed with LPC include active surveillance, radical prostatectomy, that is, surgical removal of the prostate using an open, laparoscopic or robot-assisted (robotic) procedure or radiotherapy, delivered externally using external beam or stereotactic radiotherapy or internally via brachytherapy. A recent landmark randomised controlled trial (RCT), the Prostate Testing for Cancer and Treatment (ProtecT) trial, compared treatments for LPC and found no difference in PC-specific mortality according to treatment type at a median of 10-year follow-up. ${ }^{5}$ However, the financial costs, side effects and complications for each treatment option are quite unique, ${ }^{6}$ with differing impacts on quality of life. ${ }^{37}$ For example, prostatectomy is associated with greater risk of urinary incontinence and erectile dysfunction, whereas radiotherapy is more likely to cause bowel problems. ${ }^{7}$ Subsequently, international guidelines do not indicate a single most appropriate treatment for managing LPC, instead recommending that patients be informed about all treatment options and that their preferences (and cancer severity/aggressiveness) guide decision-making. ${ }^{8}$

Recent developments in LPC treatment, such as robot-assisted prostatectomy, have further complicated patient decision-making. Robotic prostatectomies have become increasingly popular in the last decade, ${ }^{9}$ despite limited evidence of superior oncological or functional outcomes compared with other forms of prostatectomy. ${ }^{10}$ A systematic review of observational studies suggests that robotic prostatectomy results in slightly lower rates of intraoperative and perioperative complications and other adverse events than other prostatectomy types, but positive surgical margin rates appear no better than for open prostatectomy. ${ }^{11}$ Further, a recent RCT found no difference in urinary or sexual function 12 weeks after either a robotic or open prostatectomy. ${ }^{10}$ Other recently published RCTs, such as the abovementioned ProtecT trial, offer little additional insight, as only 25/391 (6\%) of the men treated with prostatectomy had a robotic procedure and no subgroup analyses were performed. ${ }^{7}$ Few studies have investigated factors underlying the uptake of robotic prostatectomy, despite the apparent disconnect between evidence and preference for robotic prostatectomy.

The lack of a single best treatment for LPC means that the most suitable treatment for an individual patient is largely dependent on their personal values regarding the consequences of treatment. While men tend to report the importance of issues such as treatment side effects, two reviews of patients' treatment choices for early stage PC show that their actual decisions do not necessarily reflect consideration of these factors. ${ }^{12}{ }^{13}$ For instance, patients who wish to avoid urinary incontinence may choose prostatectomy. It is thought that variation in treatment decisions may be more indicative of differences in both the content and methods by which patients are given information, rather than reflective of patient preferences. For instance, treatment recommendations are heavily influenced by clinician specialty, ${ }^{14}$ and treatment preferences vary based on the number and type of specialists consulted. ${ }^{15}$

\section{Decision-making outcomes in LPC}

Decision-making outcomes, including level of involvement in the decision-making, decisional conflict, decision satisfaction, treatment and cancer knowledge and decision regret have been the subject of research in men with LPC. Research suggests that men recently diagnosed with LPC experience stress and anxiety due to decisional conflict and this increases with lack of PC knowledge. ${ }^{16}$ More information about PC seems to reduce decisional conflict, ${ }^{17}$ and improve satisfaction with the decision. ${ }^{17-19}$ Higher control in the decision might be negatively associated with decisional conflict and positively related to satisfaction with decision-making. ${ }^{17}$ In addition, there is some literature that shows considerable decision regret levels after treatment for $\mathrm{LPC}^{19}{ }^{20}$ particularly because of side effects such as incontinence and impotence becoming evident with increasing physical activity post-treatment. ${ }^{21}$

Research into decision-making outcomes is expected to evolve with advances in both treatment effectiveness and range of treatment options. An increasing number of public hospitals in Australia (where treatment is provided with no out-of-pocket costs to the patient) are now offering men with LPC the choice between radiotherapy (external beam or stereotactic) and robotic prostatectomy (rather than standard prostatectomy). However, little is known about the specific information and decision-making needs of the men making this choice or the potential decision-making outcomes they experience post-treatment. Given the increased access to robotic prostatectomy and the implications of the treatment choice between radiotherapy and robotic prostatectomy for both the individual and the public health system, ${ }^{22}$ it is critical and timely that we understand the information, decision-making needs and preferences of men with LPC who are offered this choice as well as critical decision-making outcomes including involvement in decision-making, decisional conflict, decision satisfaction and decision regret.

This study will inform strategies to support men diagnosed with LPC in deciding the treatment option that best suits their needs, which may include provision of a decision aid (DA). A recently updated Cochrane review found that people exposed to DAs across a variety of healthcare contexts have generally been shown to feel more knowledgeable, better informed and clearer about their values and are likely to play a more active role in decision-making and have more accurate risk perceptions. ${ }^{23}$ However, the impact of DAs for men with LPC is less clear, with a recent systematic review of DAs specifically for LPC 
treatment choice concluding that 'scant evidence at high risk of bias suggests the variable impact of existing DAs on a limited set of decisional processes and outcomes.' (p. 239) ${ }^{24}$ Many of the DAs included in the review were found to be outdated and few appear to have specifically focused on robotic prostatectomy, as opposed to prostatectomy in general, as a treatment choice.

This study was predicated by robotic prostatectomy becoming a freely available treatment option for men treated at our institution (Liverpool Hospital). To be eligible for a robotic prostatectomy, patients have to attend a combined clinic in which they see both a urologist and a radiation oncologist before making a final decision regarding their treatment. Only patients expressing a desire for curative treatment attend the combined clinic. As a large part of participant recruitment and outcome assessment for this study centres around this clinic (see details below), we chose to specifically focus on decision-making between robotic prostatectomy and other forms of curative treatment (ie, radiotherapy). Other studies have investigated or are currently evaluating the broader choice between active surveillance, prostatectomy and radiotherapy for LPC. ${ }^{25} 26$

\section{AIMS}

The overall aim of this project is to determine how best to support men diagnosed with LPC to decide about the treatment option that best suits their needs, when robotic prostatectomy and radiotherapy are equally appropriate to offer them. The specific research questions are:

1. What is men's understanding of robotic prostatectomy versus radiotherapy as treatment options for their cancer?

2. What are men's treatment preferences and reasons for selecting one treatment over the other?

3. What information and decision support do men find helpful for making their treatment decision?

4. What are the decisional outcomes of choosing between robotic prostatectomy versus radiotherapy and are the measures used feasible and acceptable in this setting?

It is anticipated that the results of this study may be used to develop a decision-making intervention for men with LPC and evaluate its acceptability and efficacy. Hence, as part of research question (4) above the current study will quantify a range of decision-making outcomes in this sample of men deciding between robotic prostatectomy and radiotherapy to inform that potential future research, including involvement in decision-making, decisional conflict, decision satisfaction and decision regret.

\section{METHODS AND ANALYSIS}

\section{Methodological approach}

A mixed-methods study design was selected as the most appropriate, ${ }^{27}$ given the limited research specifically focusing on the choice between robotic prostatectomy and radiotherapy as active treatment options for LPC.
Using a mixed-methods approach will facilitate both breadth and depth of understanding of the issues of importance for men faced with this decision. ${ }^{27-29}$ Qualitative data will be collected via semistructured interviews ${ }^{28} 30$ with patients and via audio-taped consultations between the patient-urologist and the patient-radiation oncologist. Quantitative data will be collected from patients, through questionnaires assessing involvement in decision-making, decisional conflict, decision satisfaction and decision regret and from clinicians, with both urologists and radiation oncologists rating patients' suitability for, openness to considering and preference for robotic prostatectomy versus radiotherapy.

\section{Setting}

The study will be undertaken in one public hospital in New South Wales (NSW), Australia. Liverpool Hospital is the largest of the six hospitals in South Western Sydney Local Health District, a district with a population of over 820000 people, comprising $12 \%$ of NSW residents. The communities in this district are socially, economically, culturally and linguistically diverse and the area contributes $10 \%$ of the total new cases of cancer load in NSW. Liverpool Hospital treats more than 81000 patients annually and commenced offering the choice between robotic prostatectomy and external beam or stereotactic radiotherapy in 2017. It therefore provides the ideal setting for understanding the information, decision-making needs and preferences of men with LPC as well as critical decision-making outcomes including involvement in decision-making, decisional conflict, decision satisfaction and decision regret.

\section{Sample}

\section{Clinicians}

Urologists will be eligible to participate in this study if they have been approved to perform robotic prostatectomies at Liverpool Hospital. To date, the South Western Sydney Local Health District Medical and Dental Appointments Advisory Committee has credentialed three of the four referring urologists to perform robotic radical prostatectomies. Minimum criteria to be met prior to credentialing included a minimum total of 50 robotic radical prostatectomies previously performed as primary surgeon and an acceptable intraoperative and postoperative complication profile (ie, rates for adverse events such as rectal injury, returns to theatre and readmission, in line with internationally published Robotic Prostatectomy datasets). ${ }^{31}$ Radiation oncologists will be eligible to participate if they provide care for men with LPC at Liverpool Hospital. To date, three radiation oncologists have interacted with patients as part of the study.

\section{Patients}

Consecutive patients diagnosed with LPC and referred for treatment at Liverpool Hospital will be considered for participation. Men will be eligible to participate in this study if they: (1) have been diagnosed with primary LPC 
(ie, biopsy proven disease without evidence of nodal or distant metastatic disease), (2) have expressed a desire for curative treatment (as opposed to active surveillance), but not yet commenced treatment, (3) meet criteria for both robotic prostatectomy and radiotherapy (ie, not excluded from one treatment option due to factors such as prior complicated abdominal surgeries or pelvic abdominal irradiation, with ambiguous cases discussed at the Liverpool Hospital Genito-Urinary Multidisciplinary Team meeting), (4) are aged 18 years or older and (5) have sufficient command of the English language to complete interviews and surveys in English. Exclusion criteria include (1) geographical issues (eg, living too far away to have daily radiotherapy), (2) medical comorbidities which would preclude surgery, (3) contraindications to radiotherapy (including previous pelvic radiotherapy and active rectal inflammatory bowel disease), (4) preference for treatment in the private system, as we felt that the substantial out-of-pocket costs incurred for private robotic prostatectomies could result in different decision-making considerations among private patients.

It is anticipated that $20-30$ men will need to be interviewed before data saturation (ie, the point at which interviews reveal no new information or insights) is reached for the qualitative component of the project. While data saturation may be reached with fewer than 20 participants, recruitment will continue until a minimum of 20 participants are accrued to confirm that no new information is forthcoming and ensure adequate participant numbers for publication. This sample is also sufficient to pilot test the feasibility of using the quantitative measures, which are primarily included for exploratory purposes, to give a preliminary indication of decision-making outcomes in this population (ie, for the purpose of hypothesis generation rather than testing) and to pilot the measures for potential inclusion in potential future research evaluating a DA for patients with LPC. It is anticipated that recruitment will be open until at least the latter part of 2017 to reach a minimum of 20 participants. If resources allow, we will continue to recruit men to the quantitative component of the study to allow for a more robust exploration of quantitative decisional outcomes in this population.

\section{Recruitment}

In Australia, men with a raised prostate specific antigen or symptoms of a potential PC are referred by their general practitioner to see a urologist. Urologists will normally see patients at least twice in their private rooms, once to arrange a biopsy and second to give a diagnosis and discuss potential treatment options with patients. Participating urologists will identify potential study participants in their private Urology practices when patients return for their prostate biopsy results. When LPC is diagnosed, urologists are asked to provide sufficient information for patients to choose between active surveillance and curative treatment (ie, robotic prostatectomy or radiotherapy), but not to provide too much detail or recommend a particular type of curative treatment at this stage. Adherence to this request will be checked via an interview question asking patients what information they received from the urologist at the point of diagnosis. Patients who choose to have curative treatment will be booked into a monthly combined Urology/Radiation Oncology PC clinic (combined clinic) at Liverpool Hospital where they will be further informed about treatment with robotic prostatectomy and radiotherapy by a urologist and radiation oncologist, respectively. It is a requirement that any patient considering having a robotic prostatectomy via the public health system at Liverpool Hospital attend this newly established combined clinic to see both a radiation oncologist and a urologist before making a decision. If there is any uncertainty about a patient's suitability for both robotic prostatectomy and radiotherapy, their case will be discussed at the Genito-Urinary Multidisciplinary Team meeting to confirm their suitability and consequent eligibility for this study.

At their 'diagnosis' consultation, urologists will inform consecutive eligible patients about the study and men who express interest in the study will be contacted by a member of the research team, invited to participate and provided with a Participant Information Sheet and Consent Form. At each monthly combined clinic, research staff will also approach men who have not yet been recruited, provide them with information about the study and invite them to participate. Refer to figure 1 for a flowchart of the study process.

\section{Data collection}

Patient decision-making process and outcomes

Qualitative data regarding treatment decision-making will be collected from men with LPC through semistructured interviews. Where possible, men will be interviewed twice: first, after receiving their diagnosis of LPC from their urologist and before making a choice between active treatment options (robotic prostatectomy or radiotherapy) and second, after discussing active treatment options with a urologist and a radiation oncologist respectively at the combined clinic. Men who consent to study participation at the combined clinic will only undertake the second interview. Interviews will be conducted by a member of the research team not involved in participating patients' clinical care. Patients will have the option of completing study interviews either face-to-face or by phone and will not be financially compensated for their participation. Interviews will be transcribed verbatim and participants will be given the opportunity to review and amend the transcripts prior to inclusion in analysis. Both clinicians and patients will also be asked for permission to audio-record their consultations to gain further insight into the process of information provision and treatment decision-making. Research staff will not be present during recorded consultations. Box 1 and box 2 (see below) detail the first and second interviews, respectively.

Quantitative data will be collected from patients via a series of brief questionnaires administered in either 


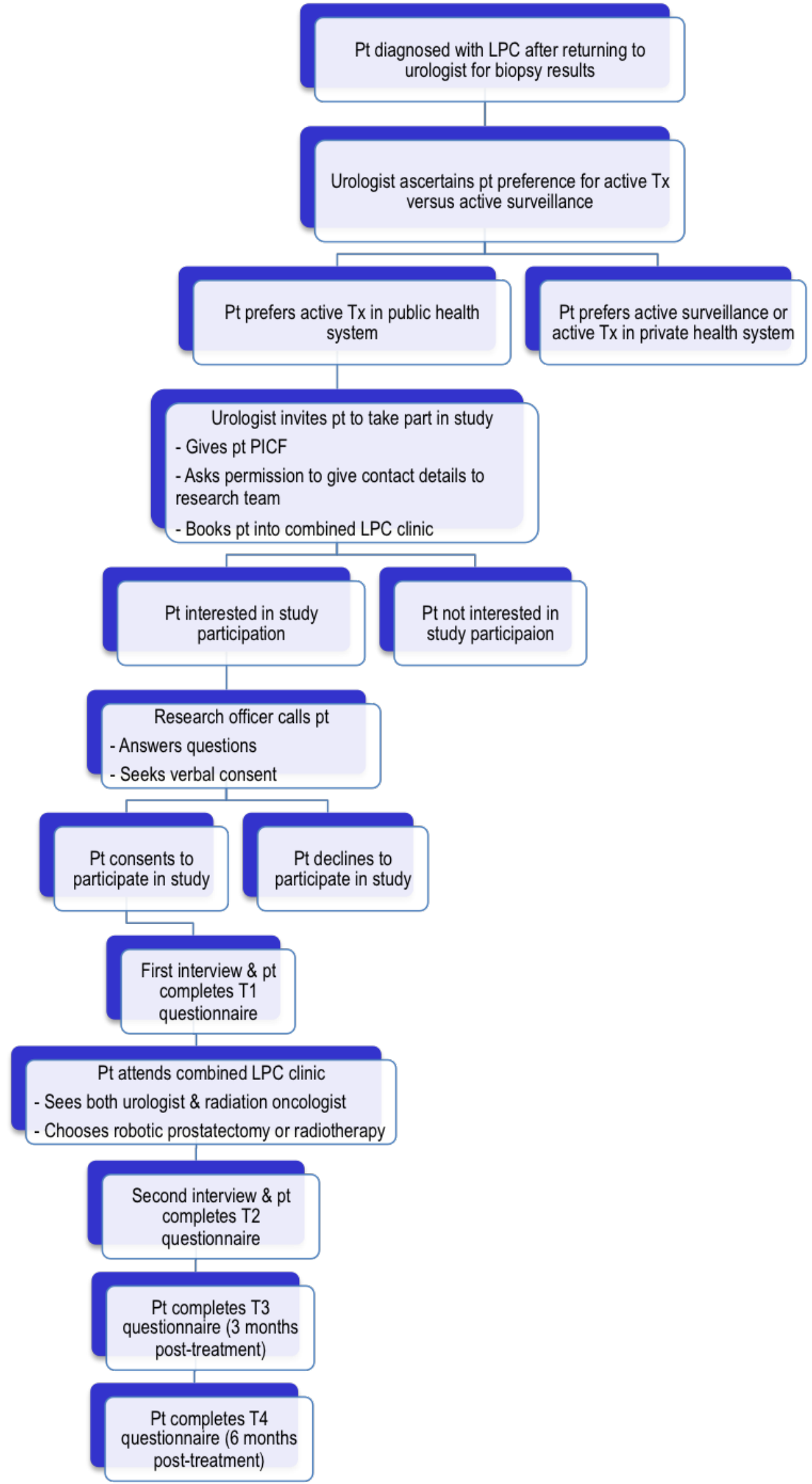

Figure 1 Study flowchart. LPC, localised prostate cancer; PICF, Participant Information Sheet and Consent Form; Pt, patient; T1, time 1; Tx, treatment. 
Box 1 First interview schedule (before attending the combined clinic)

Part A. Information provision and information needs

1. Can you please tell me a bit about how you found out you had localised prostate cancer?

2. How much did you know about prostate cancer before you were diagnosed?

3. Since your diagnosis, have you tried to find any information for yourself about treatment options?

4. If yes, what information did you seek out and how useful was the information?

- Amount (a little vs a lot, specific vs general)

- Type (clinician recommendations, statistics, patient stories)

- Format (eg, simple vs detailed, text vs audio/video)

- Source (clinicians, friends/family, internet)

5. If no, what were your main reasons for not searching out information for yourself?

6. What did your urologist tell you about your treatment options?

7. How well did you understand that information?

8. What types of questions did you ask your urologist about your diagnosis or treatment?

9. Have you talked to your General Practitioner after your prostate cancer diagnosis? Have you discussed treatment options with your General Practitioner?

\section{Part B. Treatment preferences-choice}

10. Do you currently have a preference for a particular treatment?

11. If yes, which treatment are you leaning towards? What are the reasons for preferring that particular treatment? Which one would be the main reason?

- Best chance of cure

- Minimise side effects (which particular side effects are you concerned about?)

- Maintain potency/continence

- Lifestyle or work impact

- Convenience

- Clinician recommendation

- Cost

12. If no, what are the issues that will help you to decide between treatments?

\section{Part C. Preference for involvement in decision-making}

13. Regarding how involved you want to be in selecting a treatment, do you feel that this decision is yours to make?

14. How involved would you like your partner/family to be in choosing which treatment to have?

electronic or paper form as men progress through the treatment decision-making process. The first questionnaire will be completed prior to men participating in the combined clinic. Subsequent questionnaires will be administered via paper-based or online surveys completed 1-2 weeks after making a treatment decision as well as 3 months and 6 months after treatment completion, to evaluate both short-term and long-term decisional outcomes and how these change over time. Assessment time-points were chosen based on literature regarding decisional outcomes, expected treatment outcomes and side effects in men with LPC and advice from the clinicians collaborating on the study. Table 1 details the measures included at each of the assessment time points. In addition to demographic details (age, education level, employment and marital status, country of origin, language spoken at home, previous history of cancer and previous cancer treatment), the following measures, all validated and widely used in PC decision-making research, ${ }^{24}$ were selected for inclusion in this study:

- The 16-item Decisional Conflict Scale, which assesses uncertainty in choosing options and factors contributing to uncertainty such as feeling uninformed, unclear about personal values and unsupported in decision-making. ${ }^{32}$

- The single-item Control Preferences Scale, which evaluates patients' preferred and actual involvement in treatment decision-making. ${ }^{33}$

- The 6-item Satisfaction with Decision Scale, which measures patients' satisfaction with their healthcare decisions. ${ }^{34}$

- The 5-item Decision Regret Scale, which evaluates patients' distress or remorse after a healthcare decision. ${ }^{35}$

Patient-reported qualitative and quantitative data will be supplemented by information regarding participants' disease and treatment characteristics, to be collected via medical record review.

Clinicians' assessment of patient treatment options and decisions Quantitative data will also be collected from clinicians using a study-specific measure completed by urologists and radiation oncologists immediately after seeing each patient in the combined clinic. This measure was developed in conjunction with both urologists and radiation oncologists on the study team, but its validity has not been evaluated. Relationships between clinician ratings on this measure and patient self-reported treatment preferences and choice will be evaluated as an indicator of the measure's predictive validity. A study-specific measure was used, as we were not aware of any existing validated measures to assess patients' suitability for, openness to and preferences for each treatment in this context. The measure asks clinicians for their opinion regarding:

1. A patient's suitability for both radiotherapy and robotic prostatectomy, with responses on a 4-point Likert scale: 0 'Not suitable' to 3 'Very Suitable'.

2. A patient's openness to considering the different treatment options, with 3-point Likert scale response options being: 0 'Not open', 1 'Somewhat open' and 2 'Open'.

3. A patient's treatment preference, with 5-point Likert scale response options being: 1 'Strong preference for robotic prostatectomy', 2 'Moderate preference for robotic prostatectomy', 3 'Neutral', 4 'Moderate preference for radiotherapy' and 5 'Strong preference for radiotherapy'.

4. Which treatment they consider to be most appropriate for the patient, with response options of: 'Robotic Prostatectomy', 'Radiotherapy' and 'Both Equally Appropriate'. 
Box 2 Second interview schedule (after attending the combined clinic)

\section{Part A. Information provision and information needs}

1. How was your consultation at Liverpool Hospital with the urologist and the radiation oncologist?

2. What did the urologist at Liverpool Hospital tell you about your treatment options?

- How well did you understand that information?

3. What types of questions did you ask your urologist about your treatment options?

4. What did your radiation oncologist tell you about your treatment options?

- How well did you understand that information?

5. What types of questions did you ask your radiation oncologist about your treatment options?

6. People like to receive information in different formats, such as text, audio or video available in hard copy (eg, booklet or Universal Serial Bus (USB) Flash Drive) or via the internet

- What format do you feel is the most useful for getting information about treatment options? Patient can identify more than one.

- How detailed do you think the information should be? Simple versus detailed.

- How important is it for the information to be Interactive versus non-interactive (ie, tailored vs more general).

\section{Part B. Treatment preferences-choice}

7. How did your treatment preference change after attending the combined clinic?

8. What were the reasons for choosing the treatment you did? Which one was the main reason?

- Best chance of cure

- Minimise side effects (any particular side effects you were concerned about? Impotence? Urinary incontinence? Bowel incontinence?)

- Maintain potency/continence

- Lifestyle or work impact

- Convenience

- Clinician recommendation

- Cost

9. Do you feel you had sufficient time to make your treatment decision? About how long did it take you to make your decision?

\section{Part C. Preference for involvement in decision-making}

10. How involved were you in choosing your treatment? Do you feel that this decision was yours to make?

11. How satisfied are you with your level of involvement?

12. How involved was your partner/family in choosing the treatment for your prostate cancer?

13. How satisfied are they with their level of involvement?

\section{Part D. Relationship with urologist and oncologist}

14. How would you describe your relationship with your urologist? (Helpful? Open? Inclusive?)

15. How would you describe your relationship with your radiation oncologist? (See above)

16. How important was the recommendation of your urologist and radiation oncologist in making your decision?

\section{Part E. Decisional conflict}

17. How hard was it for you to decide between the different treatments for your prostate cancer?

18. Do you feel you had enough support from others to make your choice?

19. Was there any major factor that helped you make your final choice of treatment?

\section{Part F. Decisional satisfaction}

20. How satisfied are you with your decision and why? And your partner?

\section{Data analysis}

Audio recordings of semistructured interviews will be transcribed verbatim and analysed using thematic analysis, which is 'a method for systematically identifying, organising and offering insights into patterns of meaning (themes) across a dataset'. ${ }^{36}$ Thematic analysis comprises

Table 1 Measures included at each assessment time point

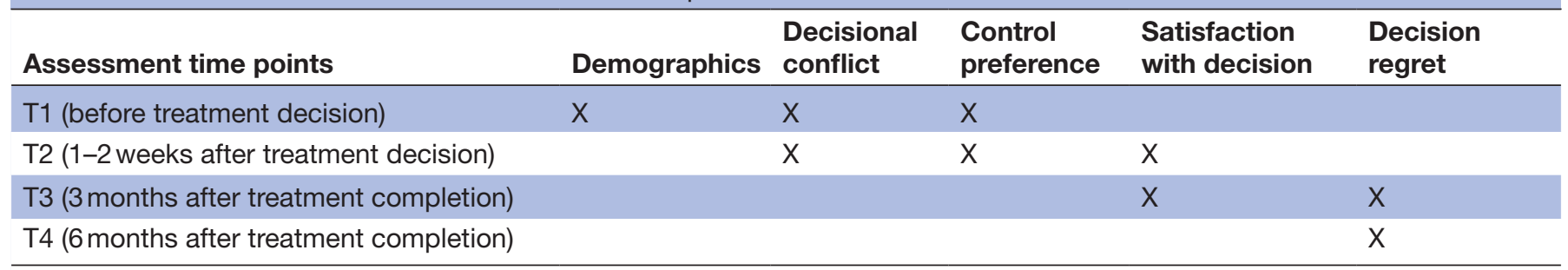

T1, time 1. 
six main phases. Transcripts will be read while listening to the audio recordings to check for accuracy and build familiarity with the data as part of the first phase. The second phase is generating initial codes, with interview transcripts being systematically coded to identify aspects of the data relevant to the research objectives. A second researcher will code a subset of interviews to ensure that the codes generated cover all interesting and relevant aspects of the data. Codes will then be collated into potential themes as part of the third phase, with themes initially reviewed by two coders as part of the fourth phase to ensure that codes within a particular theme are consistent and distinct from other themes and then checked with the broader research team. A thematic map or hierarchy will be developed in phase five via further refinement of themes and discussion of how themes fit together. Finally, a written publication of the qualitative results will be produced in phase six. While the broader research team will be consulted about the themes generated as part of phase four, the qualitative data analysis will be conducted at arms-length from members of the research team involved in the clinical care of participating patients, to avoid potential bias.

Doctor-patient consultations will be transcribed verbatim and analysed using the Observing Patient Involvement in Decision Making Scale (OPTION-5), which aims to assess how clinicians engage patients in the decision-making process during medical encounters. ${ }^{37}$ The OPTION-5 Scale (see box 3 ) is a more recent and refined version of the widely used OPTION-12 Scale ${ }^{3738}$ and has been used in oncological settings. ${ }^{39}$ The OPTION-5 Scale has demonstrated discriminative and concurrent validity and has shown acceptable levels of

\section{Box 3 OPTION-5 Scale}

1. For the health issue being discussed, the clinician draws attention to or re-affirms that alternate treatment or management options exist or that the need for a decision exists. If the patient rather than the clinicians draws attention to the availability of options, the clinician responds by agreeing that the options need deliberation.

2. The clinician reassures the patient or re-affirms, that the clinician will support the patient to become informed and to deliberate about the options. If the patient states that they have sought or obtained information prior to the encounter, the clinician supports such a deliberation process.

3. The clinician gives information or checks understanding, about the pros and cons of the options that are considered reasonable (including taking 'no action'), to support the patient in comparing the alternatives. If the patient requests clarification, explores options or compares options, the clinician supports the process.

4. The clinician makes an effort to elicit the patient's preferences in response to the options that have been described. If the patient declares their preference(s), the clinician is receptive/supportive.

5. The clinician makes an effort to integrate the patient's preferences as decisions are made. If the patient indicates how best to integrate their preferences as decisions are made, the clinician is supportive. inter-rater reliability. ${ }^{38-40}$ The OPTION-5 Scale is used by an observer (a member of the research team) to rate clinicians' behaviours that promote involvement in the decision-making process, on a 5-point scale ranging from 0 'no effort' to 4 'exemplary effort'. ${ }^{37}$ The scores from each of the five items are summed, divided by 20 and multiplied by 100 to obtain a transformed value between 0 and 100 , where 100 represents maximal effort made by the clinician to involve the patient in decision-making. ${ }^{37} \mathrm{In}$ this study, inter-rater reliability will be assessed by having two independent raters score a minimum of $20 \%$ of the consultations and calculating Cohen's Kappa coefficient.

Quantitative patient decision-making outcomes will be summarised using descriptive analyses and independent samples t-tests will be used to provide a preliminary comparison of outcomes according to treatment choice. $\chi^{2}$ tests will be used to assess differences in clinician perceptions regarding patients' suitability for, openness to and preferences for treatment according to specialty (ie, urology vs radiation oncology). While a study sample of 20-30 participants provides insufficient statistical power for more complex analyses, if we are able to continue recruiting a larger sample to the quantitative component of the study, as noted in the Patients Sample section above, we will consider multivariable analysis to control for potential moderators of decisional outcomes, such as adverse treatment effects.

\section{ETHICS AND DISSEMINATION}

The PREPaRE study adheres to the National Health and Medical Research Council National Statement on Ethical Conduct in Human Research ${ }^{41}$ and the principles of the Declaration of Helsinki. ${ }^{42}$ Potential participants will be given a participant information sheet, consent form and contact details of the research team to ask any questions about the study. They will have sufficient time to consider the invitation to participate, will be informed that study participation is entirely optional, that their decision to take part will not influence their care and that they can refuse to participate at any stage of the research even after consenting.

\section{Managing potential participant distress}

As the period shortly after diagnosis of LPC may be a difficult time for patients, a telephone script will be used when contacting patients who are considering study participation to ensure that contact with patients is conducted in a sensitive manner and to prevent causing any undue stress. Some men who agree to participate may become distressed by talking about their treatment decision-making, particularly if they are finding it hard to choose between robotic prostatectomy and radiotherapy. At the start of the study interviews, patients will be informed that they can skip any questions and/or stop the interview at any point if they find it distressing. Participants who become distressed will be offered two options to help them manage their distress. First, they will be provided with the details for the 
Cancer Council Helpline, which is a free and confidential telephone information and support service available to anybody affected by cancer in Australia. Alternatively, they will be asked whether they would like an appointment with the hospital's clinical psychologist. Participants will be informed that they will not be provided with any additional advice regarding which treatment may be most suitable for them as part of this study.

\section{Confidentiality and privacy}

Only patients' treating clinicians and project team members will be aware of whether patients are participating in the study. Alphanumeric codes assigned to participants will be used to label participant data (ie, interview audio files, transcripts and questionnaire data) in order to protect participants' identity. Names of patients and their treating clinicians will be deliberately excluded from interview and consultation transcripts as a further measure to protect participant confidentiality. Pseudonyms will be used in cases where individual data (ie, illustrative quotes) are reported in presentations or publications to ensure the privacy of participants and confidentiality of their personal data. Only aggregate quantitative data will be reported in any presentations or publications to ensure the privacy of participants and confidentiality of their data. The online questionnaire used in this study will be administered via Survey Monkey, which adheres to strict data privacy and security standards.

\section{Data storage and record retention}

All data will be stored in re-identifiable form (unique alphanumeric IDs allocated), with a list linking names and IDs kept separately. Electronic data will be stored on a password-protected computer connected to a secure network and paper data stored in a locked filing cabinet in a secure building. Data will be stored for a minimum of 7 years after publication of study results, in accordance with Institutional guidelines and the Australian Code for the Responsible Conduct of Research.

The study results will be presented at local and international seminars, conferences and published in peer-reviewed journals. All investigators will be listed as authors on any presentations or publications resulting from the study.

\section{DISCUSSION}

This study aims to determine the information, decision-making needs and preferences of men who are offered the choice between robotic prostatectomy and radiotherapy treatment for their LPC. A key strength of this research is its mixed-methods approach, with data collected from patients with LPC as well as their urologists and radiation oncologists. The audio-recorded consultations will also enable us to understand the types of information patients are both seeking and receiving during their consultations to help them make the choice between robotic prostatectomy and radiotherapy treatment for their LPC and the extent of their involvement in decision-making. Our primary focus was on the information and decision-making needs of men with LPC, but we appreciate that partners and/or family may play an important role in treatment decision-making. While we have attempted to capture partner/family levels of involvement and satisfaction in decision-making in the qualitative interviews, we acknowledge that the lack of a more in-depth exploration of partner/family influence over decision-making is a limitation of this study. The results from this study will inform how to best support men diagnosed with LPC deciding which curative treatment option best suits their needs and may identify the need for and content required in a DA to support these men.

\section{Author affiliations}

${ }^{1}$ Centre for Oncology Education and Research Translation (CONCERT), Ingham Institute for Applied Medical Research \& University of New South Wales, Liverpool, New South Wales, Australia

${ }^{2}$ South Western Sydney Clinical School, University of New South Wales, Liverpool, New South Wales, Australia

${ }^{3}$ Department of Urological Surgery, Liverpool Hospital, South Western Sydney Local Health District, Liverpool, New South Wales, Australia

${ }^{4}$ Liverpool Cancer Therapy Centre, Liverpool Hospital, South Western Sydney Local Health District, Liverpool, New South Wales, Australia

${ }^{5}$ School of Medicine, Western Sydney University, Penrith South DC, New South Wales, Australia

${ }^{6}$ Division of Surgery, Liverpool Hospital, South Western Sydney Local Health District, Liverpool, New South Wales, Australia

Acknowledgements The PREPaRE team includes clinicians and researchers from the South Western Sydney Local Health District. Their significant input is gratefully acknowledged. We are particularly grateful to Prem Rathore and Rosa Andrade for their efforts coordinating the combined clinic. We also wish to thank the patients who generously contributed their time and input this project, Helen Borger for undertaking patient interviews and Kayvan Haghighi, Nestor Lalak and Paul Gassner who conducted patient recruitment along with author Pascal Mancuso.

Contributors All authors contributed to the initial conception and design of the study. AG and DF were instrumental in securing funding for the project. ABS led the development of the initial study protocol, which AG, PM, DF and LB reviewed and provided feedback on. $A B S, O R$ and $A G$ drafted the protocol manuscript and all other authors (PM, MS, KW, MB, DF, LB) gave critical feedback. All authors read and approved the final version of the manuscript and agree to be accountable for all aspects of the work. ABS drafted the response to reviewers comments with feedback from PM, MS, KW, MB, OR, DF, LB, AG, who all read and approved the resubmitted version of the manuscript.

Funding $A B S$ and $A G$ are funded through a Cancer Institute New South Wales grant. This research was internally funded by South Western Sydney Local Health District, which has no influence on the interpretation of funding or publication of the results. This research received no specific grant from any funding agency in the public, commercial or not-for-profit sector.

Competing interests None declared.

Ethics approval South Western Sydney Local Health District Human Research Ethics Committee (HREC/16/LP00L/652).

Provenance and peer review Not commissioned; externally peer reviewed.

Data sharing statement Anyone may contact the investigators to request access to the aggregate qualitative data generated by this study. None other than the principal investigator and his team, all of whom have signed a confidentiality agreement, will have access to the audio recordings and transcripts of the interviews.

Open Access This is an Open Access article distributed in accordance with the Creative Commons Attribution Non Commercial (CC BY-NC 4.0) license, which 
permits others to distribute, remix, adapt, build upon this work non-commercially, and license their derivative works on different terms, provided the original work is properly cited and the use is non-commercial. See: http://creativecommons.org/ licenses/by-nc/4.0/

(C) Article author(s) (or their employer(s) unless otherwise stated in the text of the article) 2017. All rights reserved. No commercial use is permitted unless otherwise expressly granted.

\section{REFERENCES}

1. Australian Institute of Health and Welfare. Australian Cancer Incidence and Mortality (ACIM) books: prostate cancer. Canberra: Australian Institute of Health and Welfare, 2017.

2. Australian Institute of Health and Welfare. Cancer in Australia 2017. Canberra: Australian Institute of Health and Welfare, 2017.

3. Chen RC, Basak R, Meyer AM, et al. Association between choice of radical prostatectomy, external beam radiotherapy, brachytherapy, or active surveillance and patient-reported quality of life among men with localized prostate cancer. JAMA 2017;317:1141-50.

4. Ferrer M, Guedea F, Suárez JF, et al. Quality of life impact of treatments for localized prostate cancer: cohort study with a 5 year follow-up. Radiother Oncol 2013;108:306-13.

5. Hamdy FC, Donovan JL, Lane JA, et al. 10-year outcomes after monitoring, surgery, or radiotherapy for localized prostate cancer. $N$ Engl J Med 2016;375:1415-24.

6. Lavery HJ, Cooperberg MR. Clinically localized prostate cancer in 2017: a review of comparative effectiveness. Urol Oncol 2017;35:40-1.

7. Donovan JL, Hamdy FC, Lane JA, et al. Patient-reported outcomes after monitoring, surgery, or radiotherapy for prostate cancer. $N$ Engl J Med 2016;375:1425-37.

8. Sanda MG, Chen RC, Crispino T, et al. Clinically localized prostate cancer: AUA/ASTRO/SUO guideline. Risk 2017;6:27.

9. Shen C, Tina Shih YC. Therapeutic substitutions in the midst of new technology diffusion: the case of treatment for localized prostate cancer. Soc Sci Med 2016;151:110-20.

10. Yaxley JW, Coughlin GD, Chambers SK, et al. Robot-assisted laparoscopic prostatectomy versus open radical retropubic prostatectomy: early outcomes from a randomised controlled phase 3 study. Lancet 2016;388:1057-66.

11. Tewari A, Sooriakumaran P, Bloch DA, et al. Positive surgical margin and perioperative complication rates of primary surgical treatments for prostate cancer: a systematic review and meta-analysis comparing retropubic, laparoscopic, and robotic prostatectomy. Eur Urol 2012;62:1-15.

12. Feldman-Stewart $D$, Brundage MD, Tong $C$. Information that affects patients' treatment choices for early stage prostate cancer: a review. Can J Urol 2011;18:5998-6006.

13. Zeliadt SB, Ramsey SD, Penson DF, et al. Why do men choose one treatment over another?: a review of patient decision making for localized prostate cancer. Cancer 2006;106:1865-74.

14. Fowler FJ, McNaughton Collins M, Albertsen PC, et al. Comparison of recommendations by urologists and radiation oncologists for treatment of clinically localized prostate cancer. JAMA 2000;283:3217-22.

15. Reamer E, Yang F, Xu J. Abstract A48: Treatment decision making in a population-based sample of black and white men with localized prostate cancer. Cancer Epidemiology Biomarkers \& Prevention 2016;25(3 Suppl):A48.

16. Kaplan AL, Crespi CM, Saucedo JD, et al. Decisional conflict in economically disadvantaged men with newly diagnosed prostate cancer: baseline results from a shared decision-making trial. Cancer 2014;120:2721-7.

17. Orom $\mathrm{H}$, Biddle $\mathrm{C}$, Underwood W, et al. What Is a "Good" treatment decision? Decisional control, knowledge, treatment decision making, and quality of life in men with clinically localized prostate cancer. Med Decis Making 2016;36:714-25.

18. Berry DL, Wang Q, Halpenny B, et al. Decision preparation, satisfaction and regret in a multi-center sample of men with newly diagnosed localized prostate cancer. Patient Educ Couns 2012;88:262-7.

19. Hu JC, Kwan L, Saigal CS, et al. Regret in men treated for localized prostate cancer. J Urol 2003;169:2279-83.

20. Aning JJ, Wassersug RJ, Goldenberg SL. Patient preference and the impact of decision-making aids on prostate cancer treatment choices and post-intervention regret. Curr Oncol 2012;19:8.

21. Diefenbach MA, Mohamed NE. Regret of treatment decision and its association with disease-specific quality of life following prostate cancer treatment. Cancer Invest 2007;25:449-57.

22. Hall RM, Linklater N, Coughlin G. Robotic and open radical prostatectomy in the public health sector: cost comparison. ANZ J Surg 2014;84:477-80.

23. Stacey D, Légaré F, Col NF, et al. Decision aids for people facing health treatment or screening decisions. The Cochrane Library 2014.

24. Violette PD, Agoritsas T, Alexander P, et al. Decision aids for localized prostate cancer treatment choice: systematic review and metaanalysis. CA Cancer J Clin 2015;65:239-51.

25. Schofield P. Navigate: randomised controlled trial of an online treatment decision aid for men with localised prostate cancer and

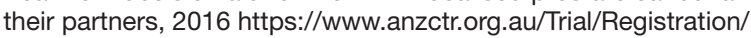
TrialReview. aspx?id=371632

26. Bayliss DR, Duff J, Stricker P, et al. Decision-making in prostate cancer-choosing active surveillance over other treatment options: a literature review. Urologic Nursing 2017;37:15-23.

27. Creswell JW. Research design: qualitative, quantitative, and mixed methods approaches: Sage publications, 2013.

28. Ross T. A survival guide for health research methods: Open University Press, 2012.

29. Tashakkori A, Teddlie C. Sage handbook of mixed methods in social \& behavioral research: Sage, 2010.

30. Breakwell GM, Hammond S, Fife-Schaw C, et al; Research methods in psychology: SAGE Publications, 2006.

31. Novara G, Ficarra V, Rosen RC, et al. Systematic review and metaanalysis of perioperative outcomes and complications after robotassisted radical prostatectomy. Eur Urol 2012;62:431-52.

32. O'Connor AM. Validation of a decisional conflict scale. Med Decis Making 1995:15:25-30.

33. Degner LF, Sloan JA, Venkatesh P. The control preferences scale. Can J Nurs Res 1997;29:21-43.

34. Holmes-Rovner M, Kroll J, Schmitt N, et al. Patient satisfaction with health care decisions: the satisfaction with decision scale. Med Decis Making 1996;16:58-64.

35. Brehaut JC, O'Connor AM, Wood TJ, et al. Validation of a decision regret scale. Med Decis Making 2003;23:281-92.

36. Braun V, Clarke V. Using thematic analysis in psychology. Qual Res Psychol 2006;3:77-101.

37. Elwyn G, Tsulukidze M, Edwards A, et al. Using a 'talk' model of shared decision making to propose an observation-based measure: Observer OPTION 5 Item. Patient Educ Couns 2013;93:265-71.

38. Barr PJ, O'Malley AJ, Tsulukidze M, et al. The psychometric properties of Observer OPTION(5), an observer measure of shared decision making. Patient Educ Couns 2015;98:970-6.

39. Stubenrouch FE, Pieterse AH, Falkenberg R, et al. OPTION(5) versus OPTION(12) instruments to appreciate the extent to which healthcare providers involve patients in decision-making. Patient Educ Couns 2016:99:1062-8.

40. Vortel MA, Adam S, Port-Thompson AV, et al. Comparing the ability of OPTION(12) and OPTION(5) to assess shared decision-making in genetic counselling. Patient Educ Couns 2016;99:1717-23.

41. National Health and Medical Research Council, Australian Research Council, Australian Vice-Chancellors' Committee. National Statement on Ethical Conduct in Human Research 2007. Canberra: National Health and Medical Research Council, 2015.

42. Declaration of Helsinki. BMJ 1964;1996:1448-9. 